\title{
La Macédoine en fanfare
}

Nicolas Prévôt

Université Paris X-Nanterre

\section{RÉSUMÉ}

Dans l'est de la Macédoine, jouant presque quotidiennement jusque dans les villages, les fanfares sont très répandues. Elles sont souvent composées de Rom, dont le répertoire emprunte alternativement à des thèmes musiciens orientaux (voire indiens) et nationaux (macédoniens). C'est en se faisant accepter comme musicien dans l'une de ces fanfares que l'auteur a pu mener cette enquête ethnomusicologique.

Mots-clefs : Musique. Fanfare. Macédoine.

Nicolas Prévôt

Musée de l'Homme

Laboratoire d'ethnomusicologie

Place du Trocadéro

75016 Paris

nicolasprevot@hotmail.com

"Ajde, narodni makedonski! Ajde, oro! Gajda, gajda!" ${ }^{1}$

Nombreux sont les villages et les villes de l'est de la République de Macédoine qui résonnent quotidiennement au son des bleh orkestari, les fanfares (les " orchestres de cuivres $\left.{ }^{2}\right)$, une tradition très répandue dans les Balkans. Au sein d'un même village, elles sont parfois plusieurs à se concurrencer pour animer la moindre fête, improvisée ou prévue de longue date, dans les alentours : réunions d'hommes au kafeana ("café ", "bar»), jours fériés, départs au service militaire ou fêtes de diplômes, mariages, ou encore circoncisions pour les communautés musulmanes rom, turques et torbes $\breve{s}^{3}$.

C'est le cas de Berovo, bourg rural de huit mille habitants situé dans la région orientale de Maleševo ${ }^{4}$, où pendant deux mois, avril et mai 1998, l'une des quatre fanfares m'a accepté au nombre de ses musiciens. La vie musicale est particulièrement intense dans ce village où les fanfares tiennent une place prépondérante aux côtés des ensembles de zurla (" hautbois»), ces deux formations offrant un répertoire traditionnel lié à la danse. À Berovo comme ailleurs, les trubac̆i comme les zurladžii ${ }^{5}$ sont toujours des $\mathrm{Rom}^{6}$. Musiciens professionnels, ils entretiennent cependant pour la plupart une activité parallèle: ils sont ouvriers, employés à l'entretien communal, forgerons.

Les bergers macédoniens, encore relativement nombreux, ont peu à peu abandonné les instruments pastoraux qui animaient les fêtes de village, principalement la gajda (" cornemuse ») dans cette région, laissant ainsi le monopole de la vie musicale aux familles de musiciens rom. Seuls les banket, les "banquets " animés en salle par des ensembles $d \check{z} e z$ tapan (" jazz tapan, batterie »), réunissent des musiciens professionnels principalement non rom, qui jouent de la musique macédonienne sur une scène avec sonorisation (batterie, guitare et basse électriques, accordéon, synthétiseur et chants).

Un premier travail monographique m'a amené naturellement à essayer de saisir la teneur du répertoire de ces ensembles de cuivres: j'ai très vite appris qu'il se résumait à trois termes, oro, ččcek, pesna, qu'il fallait cependant pouvoir discerner. Bien que jouant au sein de la fanfare à toutes les occasions et apprenant petit à petit «sur le tas" (sans compter tous les morceaux que j'avais écoutés et réécoutés depuis mon premier voyage), le sens de ces mots restait pour moi très confus. Certes, je commençais bien à aiguiser ma perception et à repérer des critères formels distinctifs, mais j'étais sans cesse dérouté par les discours contradictoires que tient la population sur cette musique.

Dans cet article je propose quelques éclaircissements sur des termes vernaculaires particuliers et je tente de montrer que la confusion peut trouver sa place dans tout système de valeurs, comme élément dynamique de construction des rapports sociaux. Je cherche à rendre compte de la nécessité et des limites d'une analyse 


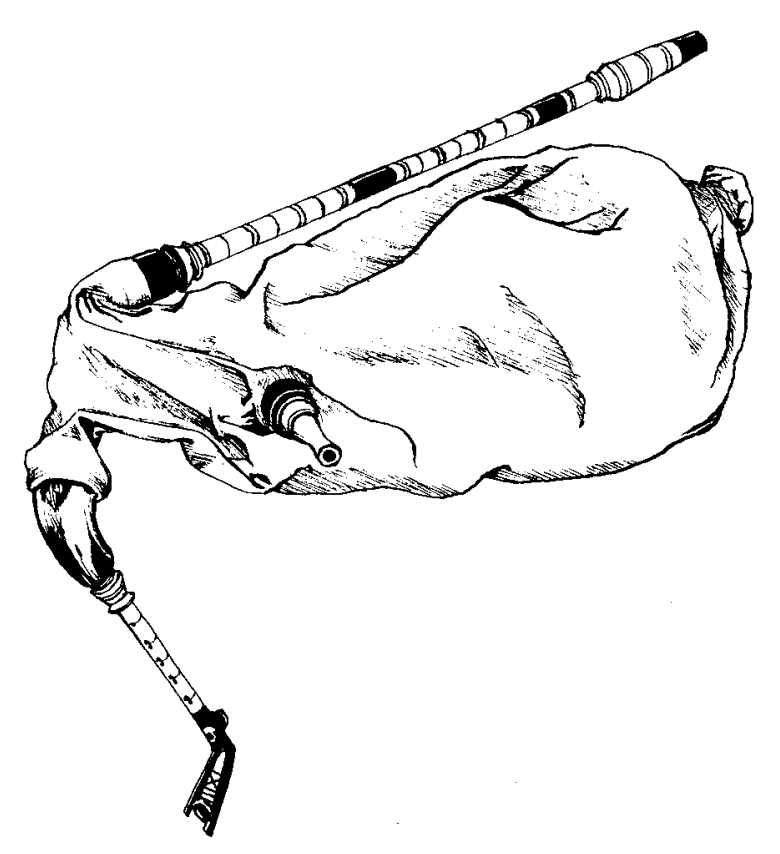

1. Gajda (dessin de l'auteur).

musicologique formelle visant à mettre au jour les différents critères de classification d'un répertoire musical, que les musiciens et les danseurs eux-mêmes ne peuvent expliquer, mais qui sont perceptibles par le biais de l'observation du processus de prestation musicale notamment.

On s'intéressera ici plus précisément aux prestations que donnent les musiciens rom aux Macédoniens, d'une part parce qu'elles sont plus fréquentes, les Macédoniens étant majoritaires, d'autre part parce qu'elles nous paraissent révélatrices des rapports qui unissent ces deux groupes, en tant que prestataires et demandeurs.

\section{- Musique et danse}

Oro, ̌̌ččk, pesna à eux trois composent l'ensemble du répertoire, mais nous écarterons d'emblée la catégorie "pesna ». Le mot signifie littéralement " chanson ", c'està-dire une mélodie liée à des paroles chantées. Cela n'empêche pas les fanfares d'en donner des versions instrumentales que l'auditoire reprend parfois en chantant. Elles sont donc facilement identifiables et ne sont pas concernées par le problème que pose la mise à jour du répertoire. D'autre part, leur base rythmico-mélodique renvoie toujours indirectement à l'un ou l'autre des deux autres termes.

Aussi réduisons-nous volontairement la classification à oro et č čček, avec la conviction qu'ils construisent à eux seuls un système ambivalent. Oro et ččcek sont d'ailleurs clairement mis en opposition sur nombre de compilations commercialisées en Macédoine sous le titre Ora $i$ сосесе ${ }^{7}$, que les morceaux soient joués par des fanfares ou par d'autres formations instrumentales ${ }^{8}$, tout simplement parce qu'ils désignent deux danses différentes. L'oro désigne une ronde ouverte alors que le ččcek est une danse individuelle. La musique des fanfares est indissociable de la danse, très présente dans la culture musicale balkanique et élément essentiel de la fête 9 .

Cette remarque devrait mettre fin à toute équivoque, mais elle annonce au contraire la première ambiguiité : la musique des fanfares est bien destinée à accompagner la danse, que ce soit avec des airs appelés « oro » ou " cočck»; mais les Macédoniens ne dansent pas le ččcek. Cependant, ils dansent bien en rondes oro sur des mélodies appelées ččcek et destinées à l'origine à la danse čččk, tout comme ils dansent en oro sur des airs oro. Il convient ici de bien distinguer les musiques appelées oro et čoček des danses auxquelles renvoient les mêmes termes. Pour les Macédoniens, le ččcek existe en tant que mélodie, mais non en tant que danse. Selon eux, la danse čc̆ek est plus proprement rom, en particulier attribuée aux femmes ${ }^{10}$. Cependant, si les Rom pratiquent le č čcek, ils dansent aussi en oro sur des airs d'oro. En revanche, l'inverse n'est pas possible : on ne peut danser en ččcek sur une mélodie appelée oro. Pour plus de clarté, voici un tableau comparant les diverses combinaisons en vigueur chez les Rom et les Macédoniens, et mettant en correspondance verticalement le ou les types de mélodie pouvant accompagner la danse.

\begin{tabular}{|l|c|c|c|}
\hline \multirow{2}{*}{ Danse } & \multicolumn{2}{|c|}{ Rom } & Macédoniens \\
\cline { 2 - 4 } & čc̆ek & oro & oro \\
\hline $\begin{array}{l}\text { Mélodie } \\
\text { associée }\end{array}$ & $\breve{c o c ̌ e k ~}$ & oro/čc̆ek & oro/čc̆ek \\
\hline
\end{tabular}

Retenons que les Macédoniens peuvent très bien danser en ronde oro, sur des mélodies oro comme sur des mélodies ččcek. La chorégraphie ne nous apporte donc aucun élément pour comprendre ce qui peut distinguer l'un de l'autre. Néanmoins, on peut imaginer logiquement que les chorégraphies oro et čoček ont imposé au départ des modèles rythmiques différents pour les mélodies correspondantes.

L'analyse musicologique de plusieurs dizaines de morceaux joués par des fanfares et nommés par les musiciens oro ou ččck, révèle que les mesures $2 / 4,4 / 4$ et $9 / 8$, les plus courantes d'ailleurs, sont communes à l'oro et au coček. En revanche, la mesure $7 / 8$ très courante est propriété exclusive de l'oro, de même pour les mesures $11 / 8$ et $5 / 8$ beaucoup plus rares ${ }^{11}$.

\begin{tabular}{|c|c|}
\hline Oro & Čočk \\
\hline $\begin{array}{lllll}4 / 4 & 7 / 8 & 9 / 8 & (5 / 8 & 11 / 8\end{array}$ & $4 / 4 \quad 9 / 8$ \\
\hline
\end{tabular}

Un certain nombre d'oro ne laissent donc aucun doute, le rythme (7/8 le plus souvent, voire $5 / 8$ ou 
11/8) suffit à les distinguer du čc̆ek. Sans qu'il sache pour autant l'expliquer, un Macédonien ne s'y trompera d'ailleurs jamais, qu'il soit musicien ou non. Pour les très nombreux morceaux binaires ${ }^{12}$ et à neuf temps, le problème reste entier et il nous faut trouver d'autres critères de distinction.

Lorsque l'on interroge les Macédoniens sur la différence qu'ils font entre oro et $\breve{c o c ̌ k}$, ils ont une réponse évidente : l'oro est "macédonien" (makedonsko), alors que le $\breve{c o c ̌ e k ~ e s t ~ p l u s ~ « o r i e n t a l » ~(o r i e n t a l n o) . ~ M a i s ~ q u e ~}$ signifie "oriental " pour un Macédonien et pour un Rom? L'analyse musicologique nous permet-elle de déceler le caractère "oriental" d'une musique ? Selon eux, le rythme autant que la mélodie contribuent à les distinguer. Nous n'entrerons pas dans une étude des échelles mélodiques qui permettrait peut-être de révéler des influences modales turques par exemple. Elle nous paraît constituer un travail à part entière qui dans le cadre de cet article n'est pas indispensable, dans la mesure où, nous le verrons plus loin, notre attention se porte sur le discours que tiennent les Rom et les Macédoniens sur la musique des fanfares. On a d'ailleurs souvent montré les influences de la culture turque dans les Balkans, en particulier chez les musiciens rom [Garfias, 1981 ; Rice, 1994]. D'autres éléments nous informent sur les origines différentes des catégories oro et ččcek.

\section{- Oro et gajda}

L'une des particularités des fanfares de la région, tellement évidente à un Macédonien qu'il faut la découvrir soi-même, est l'imitation par les clarinettistes ou les saxophonistes, voire par les trompettistes, de la cornemuse gajda. Celle-ci est souvent marquée par une formule très reconnaissable, parce que très caractéristique puisqu'elle est imposée par l'organologie particulière de la cornemuse lors du gonflement de la poche (provoquant un glissando) :

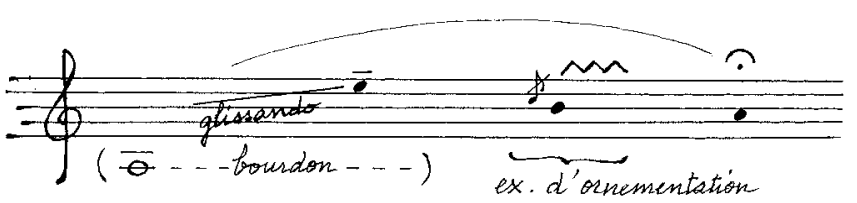

À l'appel du soliste (le saxophoniste ou le clarinettiste) qui reprend cette formule comme une annonce, tout le monde reconnaît instantanément la cornemuse, qui est associée au répertoire oro. Au-delà de cette formule presque caricaturale, la mélodie tout entière a été conditionnée par les caractéristiques de cet instrument : un ambitus d'une octave et un son continu. Il s'agit bien pour les fanfares d'interpréter des airs joués à l'origine par la gajda. En effet, on peut observer que la plupart des airs désignés comme oro évoluent à l'intérieur d'une octave; en comparaison, les ččck ont souvent un ambitus plus large.

C'est aussi par le biais des ornementations que les musiciens de fanfares reproduisent le "style " propre à la gajda. Sur le chalumeau de la cornemuse, le gajdadzia enrichit souvent les mélodies avec de simples trilles. La technique est reproduite de la même manière sur un saxophone ou une trompette, les pistons ou les clés remplaçant les trous. Le motif produit est un zigzag régulier sur un intervalle de un ton ou de un demi-ton. D'autre part, la cornemuse impose organologiquement ses contraintes à l'exécution de mélodies, qui lui conferent un style particulier, propre à l'oro. La contrainte la plus évidente est paradoxalement liée à l'avantage que procure cet instrument et qui a motivé son invention : le son continu qui est rendu possible par la poche d'air. Le musicien ne peut interrompre la mélodie pour ponctuer les phrases et doit contourner cette difficulté pour marquer une attaque entre deux notes de même hauteur qui se suivent : le fait de lever rapidement le doigt pour déboucher un trou et le reboucher aussitôt marque subrepticement une césure. Les motifs rythmiques peuvent ainsi être imprimés sur une même hauteur, en dépit d'un son continu. Ces ornementations et ces techniques, imposées par la cornemuse et travaillées comme un style par les solistes des fanfares, évoquent aussitôt la gajda à un auditeur macédonien.

Plus qu'une simple évocation, cette référence faite à un instrument d'origine pastorale ${ }^{13}$ fait figure de symbole, voire d'emblème de l'identité locale, macédonienne et plus largement slave, en opposition à celle qualifiée d'" orientale». En témoignent les réclamations fréquentes que font les danseurs aux musiciens, comme cette réaction (traduite dans la note 1) qui suivit l'interprétation d'un ččcek, lors d'un mariage : "Ajde, narodni makedonski! Ajde, oro! Gajda, gajda!» Les deux termes (oro, gajda) sont ici clairement associés et présentés comme constitutifs de l'identité macédonienne. Les trubači des fanfares cherchent alors à combler l'imaginaire et à mobiliser l'ensemble des valeurs culturelles de leur auditoire macédonien.

Les liens de l'oro et de la gajda sont certains. Au sein de la fanfare, les solistes jouent parfois à tour de rôle la partie jouée au chalumeau sur la gajda, alors que les euphoniums tiennent le "bourdon" («dem» ou "polaga $\breve{c} ")^{14}$ de la cornemuse. Mais les musiciens ne sauraient en rester là et profitent souvent de la possibilité qu'offre un tel ensemble instrumental : la basse se met alors à "arpéger ", c'est-à-dire à égrener les notes fondamentales de l'accord, en soutenant le rythme du tapan et les euphoniums se répartissent deux voix pour harmoniser le bourdon à la tierce. Le bourdon module parfois à l'appel du soliste, mais se limite à baisser de un ton puis à remonter. La polyphonie et l'harmonie restent timides, cependant l'évocation du style pastoral subsiste. Au cours d'un même morceau, les Rom cèdent souvent à la tentation de "dériver " vers une polyphonie plus 
étoffée. Notons à ce propos que la musique des fanfares et la musique des Rom en général ont tendance à faire évoluer le répertoire et son interprétation, ce que n'apprécient pas toujours les Macédoniens. Il est évident que l'on ne joue pas un oro avec un orchestre de cuivres comme on le joue avec une gajda.

Il ne s'agit pas d'affirmer que tous les oro que jouent les fanfares ont été créés à partir de la gajda, ni même que le style de la gajda y est toujours clairement identifiable (tous les oro n'évoquent d'ailleurs pas la gajda), mais la référence fréquente à la cornemuse suffit à évoquer le caractère pastoral de l'oro et à nous informer sur les valeurs qu'il mobilise auprès de l'auditoire macédonien.

\section{- Pastoral ou oriental ? Slave ou turc ?}

L'étymologie nous confirme les origines distinctes du ččcek et de l'oro. Comme le terme serbe "kolo» et le bulgare "horo", le macédonien «oro » est un mot d'ori-

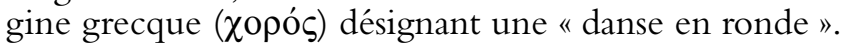
Quant au coček, le chercheur Metin And [Dunin, 1976] nous apprend qu'il est d'origine turque : dans l'empire ottoman, "un köcek était un garçon qui dansait en solo dans des fêtes entre hommes pour les divertir [...]. La musique d'accompagnement s'appelait köçekce [...]. La majorité des garçons et des filles qui dansaient étaient, en fait, des Tsiganes [...], ce qui reste de leurs danses est le çiftelli ${ }^{15}$, un type de danse improvisé ». Cette définition confirme que le čček est une danse individuelle. Le terme est souvent traduit par la dénomination générique et assez vague de « danse du ventre " (belly dance), d'ailleurs communément associée à un caractère oriental. Timothy Rice [1994 : 314] donne une définition du kyuchek en Bulgarie qui recouvre exactement nos investigations : "A Gypsy solo bellydance-like form in duple meter and in 9/8 time." (Une forme de danse tsigane en solo, proche de la danse du ventre en mesure binaire et en $9 / 8$.)

D'aucuns s'accorderont sur le caractère oriental de la musique rom de Macédoine; extraire des éléments esthétiques précis afin de mettre en évidence le "style " turc du ččck est une affaire plus délicate, d'autant qu'à l'évidence le ččcek macédonien a connu sa propre évolution depuis ses origines turques. En revanche, l'attachement des Rom macédoniens à la culture turque, indissociable de leur histoire, ne fait aucun doute. Audelà du fait qu'ils arrivèrent de Turquie avant (vraisemblablement dès la fin du XIII ${ }^{\mathrm{e}}$ siècle ${ }^{16}$ ), puis pendant la conquête des Balkans par les Ottomans, certains Rom se retrouvèrent parfois du côté de l'occupant turc parce qu'ils vivaient traditionnellement de la vente et de l'entretien des chevaux (ils étaient maquignons, forgerons, maréchaux-ferrants, comme c'est encore le cas à Berovo) et travaillèrent au service de l'armée ottomane ${ }^{17}$. Cette proximité, qui dura jusqu'à la libération tardive de la Macédoine en 1912, a notamment influencé leur langue et a conduit la majorité d'entre eux à l'Islam [Crowe, 1994 : 3] alors que les Macédoniens sont très majoritairement orthodoxes. Or, par tradition et profession, les Tsiganes reproduisent à la demande les répertoires des communautés qu'ils côtoient et pour lesquelles ils jouent. Leur musique ne peut qu'avoir été imprégnée de la culture turque, plus que ne l'a été celle des Macédoniens.

\section{- Č́č̆ek et zurla}

Si le public ne crie jamais «Zurla!» comme il lance " Gajda! " à une fanfare, c'est que zurla (" hautbois») et fanfare ne sont pas explicitement liés. En outre, il parait peu probable que les Macédoniens s'identifient à un instrument qui n'est joué que par les Rom. Nous pensons cependant que la zurla est une référence esthétique présente à l'esprit (consciemment ou inconsciemment) d'un saxophoniste ou d'un clarinettiste de fanfare, en particulier quand il joue des airs qualifiés d' "orientalno " comme les ččcek. Du fait de leurs migrations, les Rom ont apporté le répertoire oriental de la zurla ${ }^{18}$ qui symbolise leur identité en même temps qu'elle s'est enracinée depuis cinq siècles dans la vie des campagnes du sud des Balkans. Les fanfares, même si elles côtoient encore des formations de zurla, les ont parfois remplacées et ont pris leur suite dans les fêtes de village ${ }^{19}$. Ces deux formations sont d'ailleurs accompagnées par le même tambour tapan.

Nul doute que des familles de zurladžii, toujours rom, aient fait à une époque l'apprentissage de la clarinette ou du saxophone. Les zurladžii et les clarinettistes ou les saxophonistes ont d'ailleurs un lien de parenté évident à Berovo. Certains musiciens rom qui sont issus de familles traditionnellement spécialistes de la zurla semblent s'être tournés vers la clarinette, puis le saxophone. On trouve ainsi aujourd'hui des familles de zurladżii et des familles de clarinettistes saxophonistes qui ont les mêmes origines généalogiques. De même, les familles de tapandžia sont partagées entre les ensembles de zurla et les fanfares qu'ils accompagnent traditionnellement, chaque tapandžia étant en général spécialisé dans un type de formation, c'est-à-dire ensemble de zurla ou fanfare.

Ainsi les saxophonistes disent-ils préférer les saxophones altos aux ténors parce qu'ils ont moins de peine à se faire entendre dans l'aigu, mais aussi parce qu'ils aiment un son plus aigu et un timbre rappelant celui de la zurla. Le saxophone tel qu'il est joué par les Rom de Macédoine a une sonorité bien différente de celle du saxophone occidental (classique ou jazz) de même facture. Comme les clarinettistes bretons ont pris modèle sur la bombarde [Defrance, 1996], les Macédoniens ont comme référence culturelle la zurla, dont le timbre est d'ailleurs proche de celui du hautbois breton. La zurla 


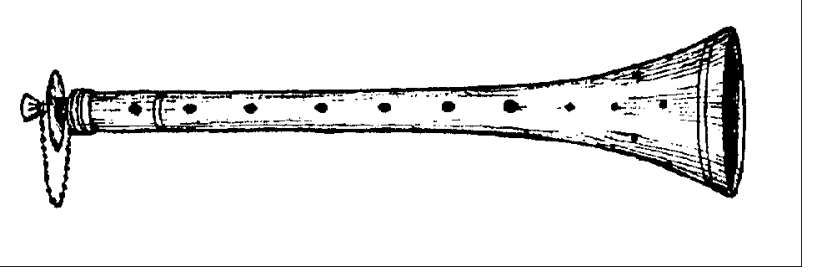

2. Zurla (dessin de l'auteur).

a imprimé un son strident et nasillard à l'esthétique musicale locale. Il est très possible que les formations de zurla et les fanfares se soient retrouvées occasionnellement à faire danser ensemble l'oro ou le ččcek.

\section{- Histoire de fanfares}

Pour bien comprendre le répertoire des fanfares, il est nécessaire de prendre en compte l'histoire de ces formations $^{20}$ : elle remonte à l'introduction dans les Balkans d'instruments occidentaux de cuivre d'origine militaire. C'est après la Première Guerre mondiale que les premières trompettes arrivent à l'est de la Macédoine de façon assez disparate à la suite du passage de troupes occidentales. Dans les petites villes et les villages, elles se mêlent d'abord à d'autres instruments, mais ne suffisent pas à créer des ensembles de cuivres, comme il y en a eu plus tôt dans la Serbie voisine, à la deuxième moitié du XIX siècle $^{21}$. Les anciens témoignent de l'existence d'ensembles hétéroclites où la trompette côtoyait la clarinette, arrivée récemment, le violon, l'accordéon et même la gajda. Mais de l'avis de tous, les cuivres étaient déjà l'appropriation des seuls Tsiganes. Les orchestres villageois réunissaient ainsi couramment des musiciens macédoniens et tsiganes, des instruments pastoraux et d'autres de facture industrielle. De rares photographies en témoignent.

Il faut attendre les années soixante pour voir se développer la pratique des cuivres chez les Rom de l'est de la Macédoine, et se formaliser progressivement des ensembles appelés bleh ${ }^{22}$ orkestari, regroupant de plus en plus exclusivement des instruments de cuivre, limeni instrumenti, jusqu'à aujourd'hui : une ou deux trompettes, un ou deux saxophones, deux ou trois euphoniums, un hélicon, un tambour (parfois un baraban, un tambour militaire) et des musiciens exclusivement rom.

Il semble que le ččcek ne soit apparu dans les villages de l'est de la Macédoine qu'avec le développement des fanfares dans les années soixante, soixante-dix. À Berovo, les anciens disent qu'il n'était pas joué avant les années soixante-dix. On peut supposer en revanche qu'il faisait déjà partie du répertoire rom mais qu'il n'était joué qu'au sein de leur communauté. Il est cependant probable qu'il était déjà connu des Macédoniens dans les grandes villes comme Skopje ou Bitola, notamment grâce aux ensembles čalgija. Le genre urbain čalgija a joué un rôle décisif dans le développement des ensembles de Macédoine, en apportant des instruments ${ }^{23}$ et un répertoire nouveaux [Linin, 1970]. D'origine turque, le čalgija était joué en Macédoine dans les kafeana, principalement par des musiciens qui étaient issus des minorités rom, juives et albanaises, et beaucoup étaient employés pour la musique de cour dans les palais des gouverneurs locaux de la Grande Porte. Très apprécié par les Turcs notamment, le čalgija n'en était pas moins considéré comme très populaire et peu d'entre eux s'abaissaient à le jouer [Rasmussen, 1996]. Dès lors, on comprend aisément que le čalgija ait influencé la musique rom en Macédoine, y compris celle des fanfares. Le rapprochement du čalgija avec le köcek dont parlait Metin And est très probable, et confirmerait à nouveau l'origine turque du ččcek.

Géographiquement et culturellement, les fanfares rom ont, semble-t-il, fait le pont entre la campagne et la ville, entre musique pastorale et musique urbaine en se développant dans des villages et des petites villes. D'un point de vue purement musical, elles semblent cristalliser toutes sortes d'influences et de rencontres. La mécanique des instruments de cuivre permettait en effet d'interpréter des répertoires divers. C'est cette polyvalence ajoutée à leur faculté de mobilité (voir note 18) qui explique en grande partie leur succès.

\section{- Le miroir de l'Orient}

Les Rom entretiennent les liens qui les unissent à la culture orientale et l'image orientale que les Macédoniens leur renvoient. Leur intérêt pour l'Orient s'explique évidemment par leurs origines et leurs migrations, mais peut-être aussi par le fait que les Rom, souvent perçus comme étrangers dans leur pays, ne peuvent s'identifier qu'à une culture étrangère.

Sur la colline de Berovo, l'ouverture des Rom sur le monde se fait par une profusion de paraboles braquées sur le soleil levant pour capter TV Asia, par exemple, ou les chaînes d'Ankara ou d'Istanbul. Les refrains mêlés des radios turques participent au bruit de fond qui n'appartient qu'à leur quartier. Les Rom sont très amateurs de variétés turques et de films indiens dont ils connaissent toutes les chansons ${ }^{24}$.

La part orientale de la culture macédonienne est laissée volontiers aux Tsiganes, qui se reconnaissent sans peine dans l'image exotique qu'on leur renvoie et affirment d'autant plus leur identité. Ces titres relevés sur des cassettes de fanfares ou d'autres musiciens rom sont révélateurs: Arapsko oro, Arapski čc̆ek ("arabe»), Derviš oro ("derviche»), Seik oro (" cheik»), Ciganski čc̆ek (" tsigane »), Romski ččce ("rom»), Esengul oro ("rose» en turc), Topaana ččcek (quartier rom de Skopje), Sutka Sutka (de "Suto Orizari » banlieue rom de Skopje ${ }^{25}$ ), etc.

Dans un article, Ljerka Rasmussen explique que l'Orient [1996; Saïd, 1978] de la Yougoslavie était 
incarné par les Rom, en particulier les Rom de Macédoine, au travers de leur musique. Autrement dit, l'Orient de l'ex-Yougoslavie était en réalité son Sud, incarné par les musiciens tsiganes macédoniens, très imprégnés de culture turque.

\section{- Derrière le miroir : l'Histoire}

Notre hypothèse est que le ččcek a eu tendance à se développer en même temps que s'est affirmé le peuple rom de Macédoine. L'oro (avec les pesna) constituait à lui seul le répertoire des fêtes de villages avant que le čc̆̌ek, amené par l'influence urbaine et les musiciens rom, ne vienne brouiller les cartes. Ce dernier a connu un nouvel essor avec celui des fanfares, et la production de cassettes a peut-être accéléré le processus. Notons encore que le développement d'ensembles formés de plus en plus exclusivement de cuivres est allé de pair avec la disparition des ensembles mixtes (musiciens macédoniens, musiciens rom) pour animer les villages ${ }^{26}$. Il ne faut cependant pas se méprendre dans l'interprétation de ce phénomène : la disparition des ensembles mixtes est due au moins autant à la disparition des musiciens de village macédoniens pour des raisons économiques qu'à une affirmation de l'identité rom. Le ččcek reste néanmoins un vecteur important de l'identité rom ${ }^{27}$.

Cette période correspond à un événement politique que nous ne devons pas négliger. En septembre 1961 se tint la Conférence de Belgrade à laquelle participait le Premier ministre indien Jawaharlal Nehru, qui, avec le président Tito, fut l'un des principaux leaders du nonalignement. Cette visite fut l'occasion de faire valoir un lien historique entre les deux pays pour affirmer l'amitié indo-yougoslave: la population rom de Yougoslavie. Des articles furent publiés dans les journaux qui évoquaient l'histoire de la migration de ce peuple nomade depuis le nord de l'Inde ${ }^{28}$. Cette histoire n'est pas une épopée racontée dans les contes tsiganes, mais une théorie scientifique confirmée depuis par des chercheurs du monde entier [Crowe, 1994 ; Fraser, 1992]. Des films populaires indiens furent projetés dans les salles yougoslaves et remportèrent beaucoup de succès. Ce fut une véritable révélation pour les communautés rom de Yougoslavie que de découvrir leurs origines indiennes de cette manière. Ils purent comprendre de nombreux mots dans les dialogues hindi et l'écran leur fit l'effet d'un miroir quand y apparurent leurs lointains " cousins ». Le film macédonien Gypsy Magic [Popov, 1997] relate avec humour ce bouleversement dans la communauté rom de Sutka à Skopje ${ }^{29}$. Les Rom sont encore les premiers amateurs de cinéma populaire indien et de leurs musiques. Aujourd'hui ils affichent la conscience de leur indianité comme une fierté. Il n'est qu'à voir les petites filles de Berovo danser le ččcek au son de la fanfare et reproduire admirablement des séries de mouvements, jusqu'aux " roulements » des yeux, qu'elles ont pu observer dans les films indiens.

Les origines indiennes ont rassemblé des communautés qui n'avaient parfois que le sentiment d'être différentes de la société environnante. Certes, les dialectes romani unissaient déjà différents groupes, mais ces groupes pour la plupart sédentaires ignoraient souvent l'existence d'une diaspora issue d'une même couche. Certains avaient déjà perdu le romani, d'autres ne se disaient même pas rom ou tsiganes, mais turcs ou égyptiens. D'autres facteurs ont contribué à l'affirmation du peuple rom : "Les Rom de Yougoslavie ont bénéficié de certains des changements mis en place dans la première moitié des années 1970 (ex. romani reconnu comme langue maternelle), qui ont renforcé leur conscience identitaire ethnique. " [Crowe, 1997 : 226-227] Il n'est donc pas étonnant d'avoir vu naître, quelques années après que les Rom ont acquis une nationalité en 1981 et juste après la chute du régime communiste, le premier parti rom de Yougoslavie en 1990 à Skopje, le PTER ${ }^{30}$, alors que les Rom n'avaient pas l'habitude de faire valoir leurs droits en tant que peuple [Silverman, 1996]. Les Rom ont aujourd'hui une Union romani internationale et ont choisi la roue indienne comme emblème.

Comment ne pas relier cette prise de conscience identitaire, suivie d'une émancipation très récente, à l'évolution de la musique rom à travers le répertoire ? Nous pensons pouvoir affirmer que les Rom ne se contentent plus d'interpréter le répertoire de la population dominante. Carol Silverman abonde dans ce sens : "Depuis les années 1970, le répertoire des Rom macédoniens s'est développé jusqu'à inclure des mélodies et chansons d'inspiration indienne, reflétant le savoir historique grandissant et la prise de conscience historique des Rom. " [1996]

Les Rom vendent leurs services à la population environnante. Celle-ci ne ferait pas appel à eux si cette musique ne lui plaisait pas, comme ils ne la joueraient pas s'ils n'y étaient pas sensibles ${ }^{31}$. Mais la musique implique ici des enjeux culturels interethniques, voire politiques. Quand les solistes des fanfares imitent la gajda, ils ne font pas qu'imiter le patrimoine musical local, mais ils se l'approprient, le jouent à leur manière et apportent une part de la culture rom au répertoire macédonien. D'une certaine manière, les Rom imposent leur culture, ou leurs références culturelles, contre les réticences des Macédoniens qui réclament que l'on ne s'écarte pas trop de la culture pastorale qui est la leur. Ceci interdit peut-être une certaine créativité et fige l'oro dans des stéréotypes que connait moins le čc̆ek.

\section{- Le $\breve{c o c ̌ e k, ~ u n e ~ f o r m e ~ " o u v e r t e " ? ~}$}

Qualifié d'abord d' "orientalna muzika", le ččcek tend aujourd'hui à accueillir des influences encore plus larges, reflétant avant tout les penchants des Rom. Il 
semble que le ččcek constitue une forme ouverte à la nouveauté, offrant sa structure musicale traditionnelle à un répertoire "étranger", exotique, oriental ou occidental ${ }^{32}$. Le terme "oriental» qui lui est donné prend alors le sens d'"exotique": variété turque (arabesk), musique considérée en Turquie comme orientale, mais aussi hymnes communistes, musiques de films populaires indiens, musiques de westerns, reprises de hits occidentaux, en particulier ceux déjà teintés d'un exotisme vendeur, comme la lambada, qui a connu un énorme succès dans les Balkans [Pettan, 1992]. Le čc̆cek est en perpétuel renouvellement tout en cultivant un caractère oriental clairement revendiqué. Il prête ses structures métrico-rythmiques et son esthétique ${ }^{33}$ à l'arrangement de mélodies empruntées à l'Orient ou à l'Occident, ou il s'en inspire de manière très créative. Si l'on y prête attention, derrière les éléments esthétiques qui donnent la couleur propre au ččcek, on reconnait parfois des mélodies insolites, comme La panthère rose, Le bon, la brute et le truand, totalement adaptées et rebaptisées à la mode locale.

Vraisemblablement, les nouvelles influences, la création de nouveaux morceaux passent bien plus par la forme $\breve{c o c ̌ c k ~ q u e ~ p a r ~ l a ~ f o r m e ~ o r o, ~ t a n d i s ~ q u e ~ l e ~ r e ́ p e r t o i r e ~}$ des fanfares laisse une place de plus en plus importante au ččcek.

\section{- La fête, entre oro et čoček}

Le succès du čoček, malgré la "résistance " de l'oro, n'est pas seulement le fruit de la persévérance des Rom à imposer leur musique, mais aussi probablement la conséquence de l'exotisme que suscite le ččcek, incarné par le musicien rom, auquel sont sensibles les Macédoniens ${ }^{34}$. Pris géographiquement entre ce que nous appelons "Orient " et "Occident ", les Macédoniens, qu'ils soient slaves ou rom, comblent leur imaginaire en se tournant vers l'un ou vers l'autre.

Lors des mariages, le šetamo ${ }^{35}$, en opposition à l'aspect collectif et régulateur des rondes oro dans lesquelles la société villageoise macédonienne exprime la cohésion, est peut-être le moment de la fête où les Rom peuvent exprimer leur identité et revendiquer leur culture à travers le ččcek. Il semble en effet que la fanfare privilégie ce dernier pour faire danser le cortège des convives. Par le šstamo s'imposeraient l'influence et la créativité rom, véhiculées par le čoček dans la société macédonienne. Mais pourquoi laisser soudain la parole aux Tsiganes, alors que l'on fait tournoyer avec fierté un grand drapeau qui revendique plus que jamais l'identité nationale macédonienne? C'est peut-être parce que les Macédoniens y trouvent eux aussi un moyen de s'exprimer, cette fois plus individuellement, par le biais d'une musique " étrangère ». En observant les Macédoniens danser sur des coček tout au long du parcours, on peut se demander dans quelle mesure ils ne se projettent pas dans un "fantasme" tsigane. Sans jamais déclarer qu'ils dansent le $\breve{c o c ̌ k}$, alcool aidant, ils esquissent pourtant quelques gestes - déhanchements, bras en l'air poignets cassés -, osent l'expression libre et s'identifient peut-être un instant à leurs voisins tsiganes en les imitant ${ }^{36}$.

Le désordre - on brise, par exemple, les bouteilles d'eau-de-vie sur le sol - et l'affirmation individuelle s'expriment pendant le šetamo et s'opposent à l'ordre et au collectif de la ronde oro, comme si un espace-temps de désordre canalisé était nécessaire à l'équilibre de la fête, elle-même métaphore de l'équilibre social. Peut-on imaginer le štamo comme une occasion formalisée d'échapper un instant à la contrainte sociale, par l'exotisme toléré du cočce ${ }^{37}$ ? C'est en tout cas le rapport complexe entre les deux communautés qui s'exprime à cet instant: en tant qu'ils représentent tout ce que la société macédonienne n'est pas, les Tsiganes gardent un statut marginal, mais l'image de liberté qu'ils inspirent fascine l'imaginaire collectif. On comprendrait dès lors pourquoi la culture rom, et le ččcek en particulier, n'est jamais totalement rejetée. Tacitement appréciée, elle semble au contraire trouver sa place dans ou par rapport à la société globale macédonienne, dans le jeu musical et l'enjeu social.

Si le caractère "oriental» de la musique rom et les influences multiples qu'elle accueille ont, semble-t-il, de plus en plus d'importance et d'influence dans le répertoire des fanfares, si le ččcek s'affirme de plus en plus dans les fêtes macédoniennes, c'est peut-être que les musiciens rom (et tout musicien professionnel en général), derrière leur apparente subordination, n'ont nul besoin d'estrade pour faire entendre une certaine supériorité, car la musique leur procure un pouvoir jamais totalement contrôlable ${ }^{38}$. Pour gagner leur vie, les musiciens doivent d'abord satisfaire leur auditoire, mais ils savent aussi imposer petit à petit leurs valeurs, même s'ils se font rappeler à l'ordre régulièrement.

Ouvrons une parenthèse pour faire référence à l'ouvrage de Gilbert Rouget, La musique et la transe. L'auteur se penche sur l'opposition faite par les Grecs anciens entre mode dorien et mode phrygien. Notre propos n'est pas celui de la transe (il ne s'agit pas non plus d'une comparaison des modes), mais ce problème offre un parallèle saisissant avec notre dialectique oro/ coček. De deux modes structurellement très différents, il ressort que le mode phrygien, s'il est communément associé à la transe (pratique dionysiaque, mania) chez les Grecs, ne le doit pas à «des faits de structure musicale " (un pouvoir sui generis propre à provoquer la transe), mais à son origine phrygienne (de Phrygie) et à ce qu'elle implique dans l'esprit des Grecs: ce mode est associé au "répertoire de transe" en référence aux valeurs qui lui sont liées, précisément comme signe de l'identité de Dionysos (Dionysos lui-même et son culte étant d'origine phrygienne). Ainsi le dorien est considéré comme "le seul mode qui soit vraiment grec " et 
moral (nous rappelant la conception que les Macédoniens ont de l'oro d'aujourd'hui), et le phrygien, bien que totalement intégré à la culture locale, comme un mode déprécié et fascinant à la fois.

Sans pousser trop loin la comparaison, on ne peut qu'être frappé par la proximité géographique des faits et surtout par l'origine orientale d'un répertoire étranger dans les deux cas : la Phrygie par rapport à la Grèce, la Turquie par rapport à la Macédoine. Notons enfin qu'en Grèce antique comme en Macédoine actuelle, le mode ou répertoire étranger (phrygien ou ččcek) est associé aux idées de débauche (le phrygien est "orgiastique " selon Aristote) et de liberté ("délivrance" dans le cas de la transe). "Le côté phrygien de l'opposition pourrait se résumer d'un mot: défoulement. " [1985: 405] Résumant Aristote, Rouget nous dit que "l'opposition dorien/phrygien subsume toute une série d'oppositions : calme/agité, viril/ non viril, digne/indigne, aristocratique/plébéien, beautél banalité, destiné à l'instruction/destiné à l'amusement». Ces oppositions rappellent avec plus ou moins de pertinence, mais de manière troublante, celles que nous nous sommes efforcés de faire apparaître pour l'oro et le ččcek. De la même manière que l'opposition dorien/phrygien, l'opposition oro/čočck s'est révélée finalement moins formelle qu'idéelle, voire idéologique, et révélatrice d'un plus large antagonisme social.

\section{- L’oro sauvegardé ?}

Dans la région de Berovo, les Macédoniens déclarent peu s'intéresser à la musique de fanfare qui est celle des Rom, cependant personne ne saurait s'en passer pour un mariage ou toute autre occasion. Les chercheurs macédoniens ne citent pas plus le čc̆ek quand ils parlent de musique macédonienne et n'y prêtent aucune attention dans leurs travaux. Le ččck est attribué aux Rom et la musique rom est un peu considérée comme une musique " étrangère".

$\mathrm{La}$ musique pastorale, volontiers reconnue comme macédonienne, a disparu dans toutes les fêtes de village. Elle ne réapparait dans les grandes villes que jouée par des ensembles folkloriques qui se réclament des tenants de la vraie tradition macédonienne. La représentation pastorale de l'oro prédomine, incompatible avec l'idée d' "ailleurs ", d'autant plus qu'il véhicule - certainement plus que jamais depuis l'indépendance du pays en 1991 l'identité nationale macédonienne. Avec mérite, les ensembles folkloriques essaient de faire vivre l'oro et la musique traditionnelle parce qu'elle ne vit plus guère dans les campagnes. Cautionnés par le gouvernement, ces spectacles qui mêlent danse et musique et que l'on exporte volontiers, participent en même temps à l'affirmation de la culture et de la nation macédoniennes. Sortie du tout social auquel elle participait et conditionnée dans un idéal de pureté, cette musique semble avoir bien du mal à se dégager du processus de standardisation et des stéréotypes. Paradoxalement, ces groupes folkloriques enferment l'oro dans une référence constante au passé, comme un musée conserve des objets.

L'anecdote suivante, concernant la Bulgarie voisine, que relate Timothy Rice offre un parallèle saisissant avec la situation macédonienne : "La musique d'ensemble [folklorique], en tant que parfait représentant de la position esthétique de l'État, était extrêmement conservatrice. Néanmoins, dans les années 1980, de plus jeunes arrangeurs et compositeurs, influencés par la popularité de la musique tsigane, commencèrent à insérer occasionnellement des éléments tsiganes dans leurs mélodies, produisant ainsi la musique pour laquelle des musiciens tsiganes avaient été punis. Cela irrita fortement les musiciens plus âgés, qui interprétaient ces efforts, non pas comme un moyen de rendre la musique populaire plus acceptable pour les amateurs de musique tsigane, mais déjà comme un nouvel échec de la part des compositeurs pour comprendre en profondeur l'essence de la musique "purement bulgare".

"Lors d'une répétition de l'ensemble folklorique de Radio Sofia en 1988, j'étais assis à côté de Stoyan Velichkov [un musicien rom], alors que les musiciens étaient en train de répéter un morceau appelé Cherkeska, d'un "nouveau compositeur". Stoyan y était ouvertement hostile, et quand je lui demandai ce qu'était ce morceau, il répondit d'un ton moqueur qu'il s'agissait d'un kyuchek, la danse solo des Tsiganes. En donnant un autre nom au kyuchek, les compositeurs, avec la caution du gouvernement, pouvaient diffuser un type de musique qui, à cause de son association avec la culture rom, était pour un temps bannie de la plupart des lieux publics. On dit qu'Ivo [Ivo Papasov, musicien rom très populaire en Bulgarie] fut même emprisonné pour l'avoir joué. Stoyan était aussi méprisant envers l'arrangement. Les airs étaient plats et disait-il: "Il n'y a rien là-dedans. Un musicien traditionnel ne ferait jamais quelque chose comme ça. C'est pour ça que les gens n'aiment pas écouter la radio."

"En référence à une mélodie particulière avec de longues tenues très peu caractéristiques de la musique bulgare, Stoyan dit: "Istanbul! Du pur turc. Mon Cherkeska est plus bulgare." Il trouvait cocasse et digne de commentaire que les turquismes interdits aux clarinettistes soient autorisés quand ils sont joués par des instruments traditionnels. De son point de vue, ou bien c'était de l'hypocrisie ou, tout simplement, les compositeurs ne savaient pas faire mieux. Ainsi, l'ensemble de la Radio, utilisant des compositeurs entrainés et approuvés, qui donnaient aux airs des noms de genres bulgares et les enrobaient d'ornements de musique occidentale, commencèrent à représenter un style qui valut à de nombreux musiciens rom la colère et les représailles des autorités. » [1994:256]

\section{- Confusion}

Il est possible de pointer ce qui différencie les deux termes en sélectionnant des exemples particulièrement contrastés, et en s'aidant du discours des informateurs 
et des remarques glanées ici et là. Mais la réalité du répertoire est tout autre. Nous constatons que l'oro et le cočck ont tendance à se confondre et à se ressembler malgré leurs divergences, et nous réalisons combien il est difficile de les distinguer. Le č ččk semble déteindre sur l'oro et réciproquement, nous laissant dans le doute pour certains airs. Le répertoire semble s'organiser comme un continuum situé entre deux pôles, illustrant par ces extrémités l'opposition sans équivoque de deux catégories musicales, voire de deux cultures, avec au milieu l'indistinction, née de la rencontre et du mélange de deux systèmes.

La frontière séparant oro et ččcek reste floue, et une analyse plus approfondie et exhaustive ne ferait probablement que prolonger cette ambiguité formelle qui semble être constitutive du répertoire. Sur le terrain, le meilleur moyen de reconnaître l'oro et le ččcek était de confronter la perception que j'avais de différents morceaux à l'interprétation qu'en faisaient les habitants. À propos d'un même morceau, j'obtenais des réponses différentes selon les interlocuteurs ou je parvenais à les faire douter, alors que les musiciens m'avaient assuré qu'il s'agissait d'un oro ou d'un ččck.

L'ambiguïté des termes oro et ččcek s'illustre par l'exemple suivant. Sur l'une des cassettes qu'elle a commercialisées, la fanfare Maleševski Melos présente le titre Firdezin ččcek: il s'agit d'une version d'un air fréquemment joué dans la région de Berovo. Firdeza est la fille de Nešat, le chef de la fanfare, à qui il est dédié. À vingt kilomètres de Berovo, j'ai eu l'occasion d'enregistrer une version du même air donnée par la fanfare du village de Crnik : elle le nomme Nevestino oro (« oro de la mariée »). On pourrait situer ce morceau (en 9/8) au milieu du continuum oro $\breve{c o c} e k$ : il ne présente pas de trait radicalement distinct comme nous avons pu en présenter. Or, à l'écoute, les Macédoniens le reconnaissaient comme un oro. Il faut préciser que les airs possèdent rarement des titres qui leur sont propres et que leur interprétation est parfois aussi libre que le titre qu'on leur donne : chaque fois, la fanfare qui l'interprète en revendique la création et se l'approprie. Dans les cas où un morceau n'est pas clairement rattaché par des critères « objectifs » à l'une ou à l'autre des catégories, on est en droit de se demander si chacune des deux communautés, rom ou macédonienne, ne se l'attribue pas en y projetant ses propres valeurs et critères sur des motifs identitaires plus ou moins conscients. Sur la confusion formelle résultant de l'influence réciproque des deux répertoires et du mélange des deux styles se grefferait ainsi la manipulation des deux communautés cherchant à s'approprier le répertoire.

Bien qu'elle nous semble très présente dans le système de valeurs que constitue le répertoire, la notion de confusion n'est apparue que tardivement dans cet exposé, parce qu'il était nécessaire de disposer des termes formels de l'opposition oro et čc̆ek pour comprendre l'ambiguiité réelle du système et les manipulations dont il est l'enjeu. S'ajoute la confusion qu'apportent les éléments esthétiques extérieurs qui rendent la compréhension du répertoire complexe. Avec l'ouverture des pays de l'Est et le développement des communications, l'actuelle variété des influences culturelles est telle qu'il est difficile de les isoler au sein d'un seul morceau. Différents styles paraissent se combiner, et les termes " oro " et " ̌coček", autrefois certainement distincts, semblent ne plus suffire à maintenir seuls la cohérence du répertoire. Certains morceaux témoignent encore sans équivoque de la réalité de l'un ou de l'autre, mais beaucoup d'autres combinent des éléments qui sont propres à chacun, comme en atteste sur une cassette ce "Svatovski čoček " ${ }^{39}$ qui présente une imitation de gajda au saxophone.

Nous avons été amenés à envisager la prestation musicale des fanfares comme un espace-temps où s'expriment les oppositions et les enjeux de deux cultures, l'une revendiquant l'identité nationale macédonienne se référant à des racines locales pastorales représentées par l'oro, l'autre cultivant son image exotique à travers le ččcek, avec ses aspirations et ses inspirations orientales qui ont été renforcées récemment par la découverte d'origines indiennes. Les fanfares rom animent aujourd'hui la vie des villages de Macédoine en réunissant ces deux aspects contradictoires ; elles jonglent avec un certain conservatisme villageois et l'accueil d'influences étrangères, deux éléments essentiels qui font de leur musique une tradition plus que jamais vivante. Musiciens professionnels, les Rom doivent séduire leur auditoire en connaissant ses valeurs et ses références esthétiques, mais, paradoxalement, ils séduisent aussi par leur différence. Une " conscience ethnique » croissante les aide à imposer cette différence contre la résistance des Macédoniens, qui pour autant ne peuvent se passer de la présence rom lors des fêtes. Aussi les fanfares et leur musique nous sont-elles apparues comme des éléments dynamiques de construction des rapports sociaux, créateurs d'ordre et de désordre, et à la fois sources d'équilibre et moteur social. 


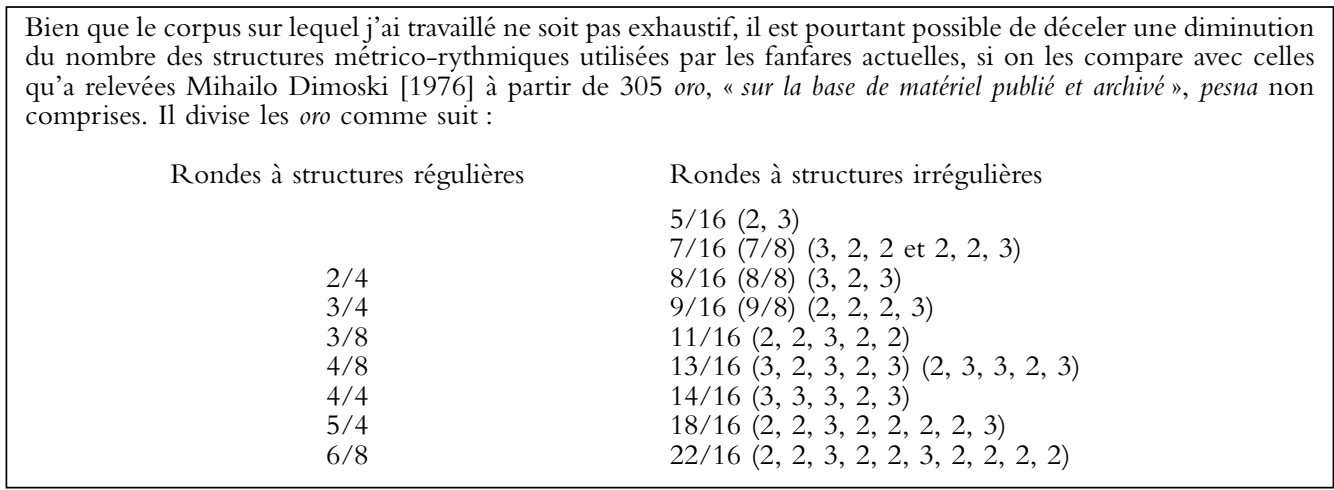

\section{Oro}

2/4 4/4 5/8 7/8 9/8 11/8 14/8

bourdon/origine monodique ambitus restreint à une octave fréquentes imitations de gajda ornementations gajda registre médium improvisations plus rythmiques improvisations plus figées

danse collective en ronde à l'arrêt (rondes) (masculin ?)

conservation socialisation

origine rurale musique pastorale oro: mot d'origine grecque macédonien

répertoire ancien et local tendance à la standardisation

\section{Čr $\breve{c k}$}

\section{$4 / 49 / 8$}

harmonisation, modulations sur plus d'une octave

référence au timbre de la zurla ornementations «orientales " registre plus étendu dans l'aigu improvisations plus mélodiques et libres improvisations moins contraintes

danse individuelle, danse du "ventre "

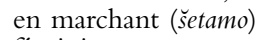

féminin

expression libre et ouverture

émancipation

influence urbaine

čalgija (origine turque)

coček : mot d'origine turque

oriental, rom, turc

influences nouvelles

« ouverture " et développement du ̌̌oček

\section{Notes}

1. «Allez, un traditionnel macédonien! Allez, un oro ! De la cornemuse, de la cornemuse! " L'assistance s'adresse à une fanfare rom, après que celle-ci a joué un ččck.

2. Bas (" hélicon»), baritoni ("euphoniums»), trubi ("trompettes»), saksofoni ("saxophones"), tapan (" tambour») : cinq à huit musiciens en moyenne.

3. Macédoniens musulmans.

4. On prononce le $\breve{s}$ «ch», le $\breve{c}$ «tch», le $\breve{z}$ « $\mathrm{j}$ ", le $j$ « y ", le $c$ «ts ».

5. Les trubači «joueurs de truba», littéralement, "trompettistes» ou, plus largement, "musiciens de fanfare", les zurladžii, "joueurs de zurla».

6. Sédentaires, les Rom de Macédoine vivent dans leurs propres quartiers, souvent situés en bordure de village.

\section{Ora $i$ с̆осесci, pluriel : "oro et ččcek".}

8. En effet, les oro et les ččck ne sont pas l'exclusivité des fanfares; ils concernent d'autres types de formations de Macédoine. Nous ne considérerons ici que les types d'oro ou de čc̆ck joués par les fanfares.

9. Les musiciens doivent connaître un répertoire très étendu pour pouvoir répondre aux demandes du public pendant plusieurs heures. Hormis les chansons et les pesna, qui se dansent aussi, les morceaux n'ont pas toujours de titres et si quelqu'un désire un air précis, il le siffle ou il le chante.

10. Ce qu'E. Dunin nous confirme : "To $m y$ knowledge the solo čc čk dance is not performed by non-Gypsies in Yougoslavia. " ("À ma connaissance, la danse en solo čččk n'est pas pratiquée par les non-Rom en Yougoslavie. ») [1973] Elle décrit trois types de danses chez les Rom de
Skopje : "The group, open circle dance (oro); processionnal walking and dancing (cetamo); the solo

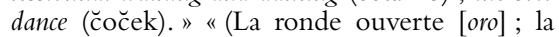
procession dansée [cetamo]; la danse soliste

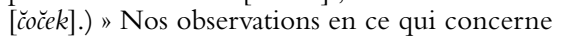
les Macédoniens rejoignent les deux premières : oro et štamo (et non cetamo).

11. E. Dunin [ibid.] décrit la danse čoček telle qu'elle a pu l'observer à Skopje, en notant qu'elle est accompagnée la plupart du temps par des rythmes en $9 / 8(2,2,2,3)$ ou $7 / 8$ (3, $2,2)$. Curieusement, en nous fondant sur le répertoire des fanfares mais aussi sur d'autres enregistrements plus largement macédoniens que nous avons comparés, les mesures $7 / 8$ caractérisent exclusivement l'oro. Il en va de même des mesures $5 / 8$ et $11 / 8$ que nous n'avons jamais relevées dans les ččcek.

12. Il nous parait délicat de différencier les morceaux à quatre temps de ceux à deux temps souvent inspirés par le kolo, une « ronde " serbe proche de la catégorie oro. 
13. La cornemuse est directement liée à l'économie pastorale et aux bergers qui les fabriquent avec les matériaux qu'ils ont à leur disposition et qui en jouent en solitaires.

14. Polaga signifie "poser».

15. Le terme çiftelli ou çiftatelli est bien connu en Turquie, mais aussi en Grèce et en Macédoine comme un rythme particulier associé à une danse. On le retrouve en Macédoine dans le répertoire des ensembles orientaux čalgija.

16. "Les Rom des pays slaves du sud et d'Albanie entrèrent dans la région à peu près de la même façon qu'arrivèrent d'autres groupes rom dans les Balkans au Moyen Âge. Leur première mention dans la région remonte à 1289 en Macédoine, bien que, comme beaucoup de rapports sur la présence de Rom dans les Balkans, ces récits furent très souvent changés en mythes [...]. Au milieu du XIV siècle, les Rom turcs s'étaient installés en Serbie, où ils étaient connus comme majstori ou maîtres artisans, grâce à leurs talents variés en matière d'art ou de commerce." [Crowe, 1994 : 195]

17. «La plupart des Rom de Skopje étaient musulmans [...]. Parmi les Rom de Macédoine les poudriers (gunpowder Gypsies), qui collectaient du salpêtre pour l'armée turque, étaient tout aussi uniques. " [Ibid. : 218]

18. On ne connait pas précisément l'origine de cet instrument mais il est très souvent joué par des Tsiganes, dans tous les pays qu'ils ont traversés depuis leur départ de l'Inde.

19. Les deux formations ont en effet l'avantage considérable de pouvoir jouer en se déplaçant, ce que réclame la tradition du šetamo, qui se déroule lors des mariages.

20. Il n'existe pas à notre connaissance d'écrits sur cette histoire; nous nous fondons donc sur l'enquête orale que nous avons menée en Macédoine.

21. La réforme des Mehterhane (orchestres des janissaires) en 1928, par laquelle les zurla ont été remplacées par des trompettes à la mode occidentale, est peut-être à l'origine de l'introduction des premiers cuivres d'Europe occidentale dans les Balkans, notamment en Serbie [Turquie, un si proche Orient, 1993].

22. De l'allemand Blechbläser (les "cuivres " d'un orchestre), pleh en serbe.

23. Instruments employés en Turquie comme la clarinette (klarinet), divers luths (lauta, tambura, saz), une cithare couchée (kanun) et un tambour (tarabuka).
24. "Macedonian Roma are particularly fond of Turkish music. " "Les Rom macédoniens sont très amateurs de musique turque. ") [Silverman, 1996]. Ce phénomène est flagrant en Macédoine, mais on peut certainement l'observer ailleurs en Europe centrale. Ainsi, en Roumanie, "many Gypsy musicians admit to listening to Radio Ankara and even to borrowing current Turkish melodies for adaptation into the muzica lautareasca. " ("Beaucoup de musiciens rom admettent écouter Radio Ankara et même emprunter des mélodies populaires turques pour les adapter à la muzica lautareasca.») [Garfias, 1981]

25. Što Orizari est l'une des plus importantes concentrations de Rom du monde (environ 45000 habitants).

26. Les ensembles mixtes n'ont pas tout à fait disparu, mais ils se limitent aujourd'hui à certains musiciens rom au sein de groupes majoritairement macédoniens jouant principalement des oro et des pesna en salle. Ces clarinettistes et ces trompettistes, les Rom étant des spécialistes de ces instruments, sont souvent connus localement et apportent certainement du prestige au groupe.

27. "Dans le cas du čoček, il semble que le principal but de sa performance soit une expression d'identité. » [Dunin, 1973]

28. Information donnée par Marcel Courtiade, professeur de langue romani à l'INALCO.

29. La bande originale, musique de Vlatko Stefanovski (avec des musiciens rom), présente notamment le titre India Dreaming.

30. PTER, parti pour l'Émancipation totale des Rom. Sont créées la même année ROMA, l'Union démocratique des Rom, et d'autres organisations politiques en Bulgarie [Crowe, 1994 : 28].

31. Si les Rom sont les seuls à jouer des cuivres en Macédoine, ces instruments doivent répondre à leurs propres critères esthétiques qu'ils ont dû imposer petit à petit à la population. Outre le timbre, le volume sonore d'une fanfare rappelle celui des couples que forment la zurla et le tapan (le hautbois zurla est beaucoup plus puissant que la gajda, relativement peu bruyante pour une cornemuse) ou celui des ensembles rom amplifiés qui atteignent un nombre record de décibels, comparés aux ensembles $d \check{z} e z$ tapan macédoniens.

32. L'adaptation d'un célèbre air de corrida sur une mesure à neuf temps et rebaptisé
Orkestarski čček par la fanfare Malesevski Melos de Berovo en est une bonne illustration.

33. Les musiciens parlent même de triler ("trilles", ornementations) propres aux styles turc, rom, grec et macédonien. Nous ne sommes cependant pas parvenus à leur faire isoler des formules caractéristiques de l'un ou de l'autre.

34. Alors que les Macédoniens voient le rêve oriental à travers les Rom, les Rom, tout en entretenant cet exotisme vendeur, voient l'Orient plus loin encore, vers l'Inde ou même vers l'Ouest.

35. Le šetamo ("promenade ") s'illustre surtout lors des mariages, qui imposent un parcours rituel allant de la maison du jeune homme à celle de la jeune femme et jusqu'à l'église. Ce cortège n'est pas considéré comme une danse à proprement parler, mais on ne peut cependant le réduire à une simple marche. Les participants esquissent des mouvements et marchent en rythme sur des pas plus ou moins inspirés des oro. Mais il s'apparente à une danse individuelle et beaucoup moins formelle que la ronde de l'oro, et il n'est pas sans évoquer le coček.

36. Entre la virilité des hommes qu'exprime le sérieux de l'oro et la féminité qui se dégage de la danse individuelle du šetamo, le contraste est parfois frappant. Même si les attitudes sont beaucoup plus retenues et l'allusion moins évidente, on ne peut s'empêcher de penser aux fêtes du Maghreb et du Mashrek, où les hommes dansent entre eux en imitant les femmes.

37. «La tâche principale du musicien tsigane est de fournir un produit musical qui ne peut être reconnu comme "tsigane" et permet à l'audience de s'identifier à "l'esprit tsigane". [...] En d'autres termes, c'est la tâche du musicien tsigane de faire vivre la part de tsigane intériorisée chez les Serbes. " [Van de Port : 180-181] Van de Port, en montrant l'importance que peut avoir la musique rom dans les kafeana comme processus d'identification et comme exutoire à la violence, suggère que les musiciens tsiganes puissent être l'objet d'une projection imaginaire. "À l'intérieur des bars tsiganes, la porte est ouverte à toutes ces choses interdites et cachées qui étaient déposées sous la figure du Tsigane. " [Ibid. : 188]

38. Le gouvernement bulgare en est même venu à interdire le ččce [Rice, 1994].

39. Maleševski Melos. C̆očeci, Skopje, Mister Kompani Ltd.

\section{Références bibliographiques}

BRANDL, 1996, "The "Yiftoi" and the Music of Greece. Role and Function", The World of Music, 1.

BuChanan Donna A., 1995, "Metaphors of Power, Metaphors of Truth: The Politics of Music Professionalism in Bulgarian Folk Orchestras ", Ethnomusicology, 39 (3) : 381.
Crowe David M., 1994, A History of the Gypsies of Eastern Europe and Russia, New York, Saint Martin's Press.

Defrance Yves, 1996, "Musiques traditionnelles de Bretagne. Sonnoux et sonerien ", Skol Vreizh, 35.

Dimoski Mihailo, 1976, «Les structures métrico-rythmiques des rondes en Macédoine ", Makedonski Folklor, 18. 
Dunin Elsie, 1971, "Gypsy Wedding : Dance and Customs ", Makedonski Folklor, 7-8.

- 1973, "Coček as a Ritual Dance among Gypsy Women ", Makedonski Folklor, 12.

Fraser Angus, 1992, The Gypsies, Oxford, Blackwell.

GARFIAS Robert, 1981, "Survival of Turkish Characteristics in Romanian Musica Lautareasca ", Yearbook of the International Folk Music Council, 13 : 97-107.

Gojkovic Andriana, 1986, "Music of the Yougoslavian Gypsies ", Traditional Music of Ethnic Groups Minorities : 187-193.

GOLABOCKI Sotir, 1974, «Potekloto na zurlata ( "L'origine de la zurla »), Makedonski Folklor, 13.

JovANOVIC Jarco, 1996, « Les Tsiganes en Serbie », Études tsiganes, $1-2$.

LiNIN Aleksandar, 1970, "Instrumentalnite sostavi vo Makedonskata narodnaMuzika " "Les ensembles instrumentaux dans la musique populaire macédonienne "), Makedonski Folklor, 5-6.

- 1986, Narodnite muzički instrumenti vo Makedonija ("Les instruments de musique traditionnels en Macédoine "), Skopje, Makedonske Kniga.

LORTAT-JACOB Bernard, 1987, L'improvisation dans les musiques de tradition orale, Paris, SELAF.

- 1994, Musiques en fête : Maroc, Sardaigne, Roumanie, Nanterre, Société française d'Ethnologie.

Pejoska Frosa, 1989, «Comptes rendus sur des textes de Veronika Görög », Cahiers de Littérature orale, 25 : 226-234.

- 1998, "Les musulmans de Macédoine », 2 conférences données à l'INALCO.

Pettan Svanibor, 1992a, «Selling Music. Rom Musicians and the Music Market in Kosovo ", Echo der Vielfalt. Echoes of Diversity : 233-245.

- 1992b, "Lambada in Kosovo : A Profile of Gypsy Creativity ", Journal of the Gypsy Lore Society, 2 : 117-130.

- 1996, "Gypsies, Music and Politics in the Balkans. A Case Study from Kosovo ", The World of Music, 1.
Popov Stole, 1997, Gypsy Magic, Skopje, Vardan Film.

RASMUSSEN Ljerka, 1991, "Gypsy Music in Yougoslavia : Inside the Popular Culture Tradition ", Journal of the Gypsy Lore Society, Series 5, 1 (2) : 127-139.

- 1996, "Orientalism, Rom Gypsy, and the Culture at Intersection ", Echo der Vielfalt. Echoes of Diversity : 247-254.

RICE Timothy, 1982, "The Surla and Tapan Tradition in Yougoslavian Macedonia ", The Galpin Society Journal : 117-137.

- 1993, "A Macedonian Sobor: Anatomy of a Celebration", Journal of American Folklore, 368.

- 1994, May it Fill your Soul : Experiencing Bulgarian Music, Chicago, Chicago University Press (+ CD).

Rouget Gilbert, 1980, La musique et la transe, Paris, Gallimard (coll. «Tel»).

SAÏD Edward W., 1980 (1978), L'orientalisme. L'Orient créé par l'Occident, Paris, Seuil.

Silverman Carol, 1976, "Les Tsiganes en Serbie ", Études tsiganes, $1 / 2: 9-15$.

-1988a, "Negotiating "Gypsiness" Strategy in Context ", Journal of American Folklore, 101 : 261-275.

- 1988b, "Tsiganes, musique et politiques en Bulgarie », Études tsiganes, $4: 14-20$.

- 1996, "Music and Power: Gender and Performance among Roma of Skopje, Macedonia ", The World of Music, 1.

TAssy Martine, 1993, "Poètes rom de Macédoine et du Kosovo ", Ethnies, 15 : 122-131.

TODOROV Wassil, 1994, "Histoire des cuivres en Bulgarie », Brass Bulletin, 88 (4) : 72-76.

Turquie, un si proche Orient, 1993, Argenteuil, Mairie d'Argenteuil : 131-135.

VAN De PORT Mattijs, 1998, Gypsies, Wars and Other Instances of the Wild. Civilisation and its Discontents in a Serbian Town, Amsterdam, Amsterdam University Press.

Williams Patrick, 1993, "Chanter la séparation. Musique et identité », Ethnies, 15 : 139-144.

\section{ABSTRACT}

\section{Brass bands in Macedonia}

Brass bands are very common in the east of Macedonia and play daily in the villages. They are often composed of Romanies whose repertory is inspired both by oriental (and even Indian) and national (Macedonian) musical themes. It is as a musician admitted into one of these brass bands that the author could make his ethnomusicological investigation.

Keywords: Music. Brass band. Macedonia.

\section{ZUSAMMENFASSUNG}

\section{Blaskapellen in Makedonien}

Blechmusik ist im westlichen Makedonien weitverbreitet und fast jeden Tag in Dörfern gespielt. Blaskapellen sind oft aus Zigeunern zusammengesetzt, deren Repertoire abwechselnd von orientalischen (und eben indischen) und nationalen (makedonischen) musikalischen Themen entnommen werden. Der Autor hat sich als Musiker in einer dieser Blaskapellen annehmen lassen, um seine ethnomusikologische Untersuchung durchzuführen.

Stichwörter: Musik. Blaskapelle. Makedonie. 